\title{
Effects of Mangifera pajang Kostermans juice on plasma antioxidant status and liver and kidney function in normocholesterolemic subjects
}

\begin{abstract}
The effects of a bambangan juice powder (BJP) drink on plasma vitamin and antioxidant enzyme levels and liver and kidney function were investigated. Thirty-two healthy subjects (12 male and 20 female) ages 24-28 years were recruited from the Faculty of Medicine and Health Sciences of University Putra Malaysia, Malaysia. Compared with consuming the placebo, consumption of the BJP drink daily for 9 weeks significantly increased the concentration of plasma $\beta$-carotene and ascorbic acid. Plasma total antioxidant status was increased, but liver and kidney functions were unaffected after consumption of the BJP drink. The consumption of a BJP drink resulted in a significant improvement in certain cardiovascular biochemical parameters and thus reduced the risk of cardiovascular disease.
\end{abstract}

Keyword: Mangifera pajang; Total antioxidant status; Enzymes; Function test 